\title{
Diffusive mass transfer by nonequilibrium fluctuations: Fick's law revisited
}

\author{
Doriano Brogioli \\ Dipartimento di Fisica and Istituto Nazionale per la Fisica della Materia (INFM), Università degli Studi di Cagliari Strada Provinciale \\ Monserrato-Sestu km 0,7, 09042 Monserrato (CA), Italy
}

Alberto Vailati

Dipartimento di Fisica and Istituto Nazionale per la Fisica della Materia (INFM), Università degli Studi di Milano, via Celoria 16, 20133 Milano, Italy

(Received 19 May 2000; published 22 December 2000)

\begin{abstract}
Recent experimental and theoretical works have shown that giant fluctuations are present during diffusion in liquid systems. We use linearized fluctuating hydrodynamics to calculate the net mass transfer due to these nonequilibrium fluctuations. Remarkably, the mass flow turns out to coincide with the usual Fick's one. The renormalization of the hydrodynamic equations allows us to quantify the gravitational modifications of the diffusion coefficient induced by the gravitational stabilization of long wavelength fluctuations.
\end{abstract}

DOI: 10.1103/PhysRevE.63.012105

PACS number(s): 05.40.-a, 05.60.-k, 11.10.Gh, 66.10.Cb

Recent experiments have shown that giant nonequilibrium concentration fluctuations are a universal feature of diffusion in binary liquid mixtures and macromolecular solutions $[1,2]$. The presence of long-range correlations in a diffusing liquid mixture is a surprising result, as diffusion is usually thought to give rise to a homogeneous mixing at the molecular level, other length scales playing no role in the process. The results of these experiments have been interpreted by means of an extension of Landau's fluctuating hydrodynamics [3] to time-dependent diffusion processes [4].

Linearized hydrodynamics shows that the fluctuations are generated by thermal velocity fluctuations, due to the presence of a macroscopic nonequilibrium concentration gradient. The mechanism is analogous to that first predicted $[5,6]$ and then observed [7-10] for stationary thermal diffusion in a binary liquid mixture. Velocity fluctuations parallel to the macroscopic gradient displace parcels of fluid into regions with different concentration, thus giving rise to concentration fluctuations [11]. Since the fluctuations displace mass along the concentration gradient, one might wonder if they contribute significantly to the macroscopic diffusive mass flow.

We will show that, quite unexpectedly, the net mass transfer due to nonequilibrium fluctuations corresponds to the macroscopic Fick's mass flow. Traditionally, the diffusive flow has been interpreted as due to the random hops of the molecules in the fluid. Here we show that Fick's flow is due to random hops of parcels of fluid whose dimensions span all the length scales from the molecular up to the macroscopic one. This is a surprising result, as usually thermal fluctuations are thought of as small perturbations of a macroscopic state, while here we show that the macroscopic flow is determined by a second-order effect in the fluctuations. The introduction of new degrees of freedom, the hydrodynamic fluctuations, determines an ultraviolet divergence of the diffusion coefficient, which we regularize by imposing a cutoff at large wave vectors. The regularization procedure involves the introduction of an arbitrary length scale. By imposing that the cutoff length scale is of the order of the correlation length of the mixture, we find that the diffusion coefficient displays the usual Stokes-Einstein form and is of the same order of magnitude as the phenomenological one. However, the introduction of an arbitrary length scale into the theory can be avoided by a renormalization of the hydrodynamic equations. The renormalization procedure allows us to quantify the modifications of the phenomenological diffusion coefficient due to the gravitational stabilization of long wavelength fluctuations.

We will now derive the net contribution of the nonequilibrium fluctuations to the mass transfer. The sample considered is a binary mixture of concentration $c(\mathbf{x}, t)$ under rest. The mixture is in a macroscopic nonequilibrium condition due to the presence of a homogeneous and stationary concentration gradient $\nabla c$. A local fluctuation in the current of mass can be written as the second order product

$$
\delta \mathbf{j}_{f}(\mathbf{x}, t)=\rho \delta c(\mathbf{x}, t) \delta \mathbf{u}(\mathbf{x}, t),
$$

where $\delta c(\mathbf{x}, t)$ is the local concentration fluctuation and $\delta \mathbf{u}(\mathbf{x}, t)$ the local velocity fluctuation. Equation (1) shows that a current fluctuation takes place whenever a concentration fluctuation and a velocity fluctuation occur simultaneously at the same place.

The net contribution of the fluctuations to the diffusive current is obtained by averaging over the configurations of the system:

$$
\mathbf{j}_{f}(\mathbf{x}, t)=\rho\langle\delta c(\mathbf{x}, t) \delta \mathbf{u}(\mathbf{x}, t)\rangle .
$$

At thermodynamic equilibrium fluctuations in the velocity and concentration are not correlated. Therefore, the probability of a current fluctuation is small and with random sign, and the net flow $\mathbf{j}_{f}$ averages to zero. Under nonequilibrium conditions, however, velocity fluctuations are correlated to concentration fluctuations [4-6]. The presence of a macroscopic concentration gradient determines the existence of velocity-induced concentration fluctuations: a velocity fluctuation determines a small vortex inside the fluid and this brings parcels of fluid with a certain concentration into layers of fluid with a different concentration. On the contrary, the gravity force determines the opposite mechanism: whenever a concentration fluctuation is produced, the buoyancy force 
tends to displace the fluctuation. Therefore, terms in the average of Eq. (2) are correlated and this determines a nonvanishing mass flow $\mathbf{j}_{f}$.

We will shortly derive an explicit expression for $\mathbf{j}_{f}$ by determining the cross-correlation properties of concentration and velocity fluctuations in the presence of a macroscopic concentration gradient. This task can be accomplished by using Landau's linearized hydrodynamics [3]. Basically, one writes down the relevant hydrodynamic equations for a binary mixture. The equations are then linearized for small fluctuations of the hydrodynamic variables around their macroscopic values and random source terms are added to them.

The equations thus obtained for a binary mixture where a uniform concentration gradient is present are [4]

$$
\begin{aligned}
\delta c(\mathbf{q}, \omega)\left(i \omega+D_{0} q^{2}\right)= & -\delta \mathbf{u}(\mathbf{q}, \omega) \cdot \nabla c+i \mathbf{q} \cdot \mathbf{F}(\mathbf{q}, \omega), \\
\delta \mathbf{u}(\mathbf{q}, \omega)\left(i \omega+\nu q^{2}\right)= & \beta \delta c(\mathbf{q}, \omega)\left[\mathbf{g}-\mathbf{q}\left(\mathbf{q} \cdot \mathbf{g} / q^{2}\right)\right] \\
& +(i / \rho) \mathbf{q} \wedge \mathbf{S}(\mathbf{q}, \omega),
\end{aligned}
$$

where $D_{0}$ is the bare diffusion coefficient, $\nu$ the kinematic viscosity, $\mathbf{g}$ the gravitational acceleration, $\beta=\rho^{-1}(\partial \rho / \partial c)$, and $c(\mathbf{q}, \omega)$ is the space-time Fourier transform for the concentration fluctuations defined by

$$
\delta c(\mathbf{q}, \omega)=\frac{1}{(2 \pi)^{4}} \int e^{i \mathbf{q} \cdot \mathbf{x}+i \omega t} \delta c(\mathbf{x}, t) d \mathbf{x} d t,
$$

and an analogous expression holds for the velocity fluctuations.

In Eq. (3) $\mathbf{F}(\mathbf{q}, \omega)$ and $\mathbf{S}(\mathbf{q}, \omega)$ represent random source currents for concentration fluctuations and velocity fluctuations, respectively. As customary, their correlation properties are assumed to retain their equilibrium values [12], as a local equilibrium assumption is known to adequately represent them [13]:

$$
\begin{gathered}
\left\langle F_{i}(\mathbf{q}, \omega) F_{j}\left(\mathbf{q}^{\prime}, \omega^{\prime}\right)\right\rangle \\
=\delta_{i j} \delta\left(\mathbf{q}+\mathbf{q}^{\prime}\right) \delta\left(\omega+\omega^{\prime}\right) \frac{K_{B} T}{8 \pi^{4}} \frac{D_{0}}{\rho}\left(\frac{\partial c}{\partial \mu}\right)_{p, T}, \\
\left\langle S_{i}(\mathbf{q}, \omega) S_{j}\left(\mathbf{q}^{\prime}, \omega^{\prime}\right)\right\rangle=\delta_{i j} \delta\left(\mathbf{q}+\mathbf{q}^{\prime}\right) \delta\left(\omega+\omega^{\prime}\right) \frac{K_{B} T}{8 \pi^{4}} \nu \rho, \\
\left\langle F_{i}(\mathbf{q}, \omega) S_{j}\left(\mathbf{q}^{\prime}, \omega^{\prime}\right)\right\rangle=0 .
\end{gathered}
$$

In Ref. [4] the linearized equations (3) were used to derive the static and dynamic structure factor of the fluctuations. In the present work we will use them to derive the mass flow.

Let us briefly comment on Eq. (3). The left terms specify that concentration and velocity fluctuations decay with lifetimes $1 / D_{0} q^{2}$ and $1 / \nu q^{2}$, respectively. The right terms are source terms for the fluctuations. Beyond the random currents $\mathbf{F}(\mathbf{q}, \omega)$ and $\mathbf{S}(\mathbf{q}, \omega)$, they contain the cross coupling between velocity and concentration fluctuations. The crosscoupling term in the concentration equations shows that a velocity fluctuation $\delta \mathbf{u}$ couples with the macroscopic concentration gradient $\boldsymbol{\nabla} c$, thus giving rise to a concentration fluctuation. The cross-coupling term in the velocity equation represents the acceleration acting on a concentration fluctuation due to the buoyancy force. We have assumed that the fluid is incompressible; therefore the acceleration acting on the fluctuation is the component of gravity perpendicular to the wave vector $\mathbf{q}$.

The explicitation of $\delta c$ and $\delta \mathbf{u}$ in Eq. (3) yields

$$
\delta c(\mathbf{q}, \omega)=\frac{i\left(i \omega+\nu q^{2}\right) \mathbf{q} \cdot \mathbf{F}(\mathbf{q}, \omega)-(i / \rho) \mathbf{q} \wedge \mathbf{S}(\mathbf{q}, \omega) \cdot \nabla c}{\left(i \omega+D_{0} q^{2}\right)\left(i \omega+\nu q^{2}\right)+\beta\left[\mathbf{g}-\mathbf{q}\left(\mathbf{q} \cdot \mathbf{g} / q^{2}\right)\right] \cdot \nabla c}
$$

and

$$
\begin{aligned}
\delta \mathbf{u}(\mathbf{q}, \omega)= & \frac{i \mathbf{q} \cdot \mathbf{F}(\mathbf{q}, \omega) \beta\left[\mathbf{g}-\mathbf{q}\left(\mathbf{q} \cdot \mathbf{g} / q^{2}\right)\right]+(i / \rho)\left(i \omega+D_{0} q^{2}\right) \mathbf{q} \wedge \mathbf{S}(\mathbf{q}, \omega)}{\left(i \omega+D_{0} q^{2}\right)\left(i \omega+\nu q^{2}\right)+\beta\left[\mathbf{g}-\mathbf{q}\left(\mathbf{q} \cdot \mathbf{g} / q^{2}\right)\right] \cdot \nabla c} \\
& +\frac{(i / \rho)\left[\beta / i \omega+\nu q^{2}\right]\left\{\mathbf{q} \wedge \mathbf{S}(\mathbf{q}, \omega) \boldsymbol{\nabla} c \cdot\left[\mathbf{g}-\mathbf{q}\left(\mathbf{q} \cdot \mathbf{g} / q^{2}\right)\right]-\mathbf{q} \wedge \mathbf{S}(\mathbf{q}, \omega) \cdot \boldsymbol{\nabla} c\left[\mathbf{g}-\mathbf{q}\left(\mathbf{q} \cdot \mathbf{g} / q^{2}\right)\right]\right\}}{\left(i \omega+D_{0} q^{2}\right)\left(i \omega+\nu q^{2}\right)+\beta\left[\mathbf{g}-\mathbf{q}\left(\mathbf{q} \cdot \mathbf{g} / q^{2}\right)\right] \cdot \boldsymbol{\nabla} c} .
\end{aligned}
$$

We are now in the position to evaluate the mass flow $\mathbf{j}_{f}$. In the reciprocal space Eq. (2) becomes

$$
\mathbf{j}_{f}=\rho \int\left\langle\delta c(\mathbf{q}, \omega) \delta \mathbf{u}\left(\mathbf{q}^{\prime}, \omega^{\prime}\right)\right\rangle d \mathbf{q} d \mathbf{q}^{\prime} d \omega d \omega^{\prime},
$$

where an exponential factor $\exp \left[i\left(\mathbf{q}+\mathbf{q}^{\prime}\right) \cdot \mathbf{x}+i\left(\omega+\omega^{\prime}\right) t\right]$ has been omitted imposing $\mathbf{x}=0$ and $t=0$, as we are considering a homogeneous and stationary system.

The integrand in Eq. (10) can be decomposed as the sum of terms containing correlations of the random currents $\mathbf{F}$ and $\mathbf{S}$. In the evaluation of the integral of Eq. (10) the contribution of the second term of Eq. (9) vanishes. Due to (7) the only nonvanishing terms are those originated from self correlations:

$$
\begin{aligned}
\left\langle\delta c(\mathbf{q}, \omega) \delta \mathbf{u}\left(\mathbf{q}^{\prime}, \omega^{\prime}\right)\right\rangle= & \left\langle\delta c(\mathbf{q}, \omega) \delta \mathbf{u}\left(\mathbf{q}^{\prime}, \omega^{\prime}\right)\right\rangle_{F} \\
& +\left\langle\delta c(\mathbf{q}, \omega) \delta \mathbf{u}\left(\mathbf{q}^{\prime}, \omega^{\prime}\right)\right\rangle_{S}
\end{aligned}
$$

where 


$$
\begin{aligned}
&\left\langle\delta c(\mathbf{q}, \omega) \delta \mathbf{u}\left(\mathbf{q}^{\prime}, \omega^{\prime}\right)\right\rangle_{F} \\
&= \delta\left(\mathbf{q}+\mathbf{q}^{\prime}\right) \delta\left(\omega+\omega^{\prime}\right) \frac{K_{B} T D_{0}}{8 \pi^{4} \rho}\left(\frac{\partial c}{\partial \mu}\right)_{p, T} \beta \\
& \times \frac{q^{2}\left(i \omega+\nu q^{2}\right)\left[\mathbf{g}-\mathbf{q}\left(\mathbf{g} \cdot \mathbf{q} / q^{2}\right)\right]}{\mid\left(i \omega+D_{0} q^{2}\right)\left(i \omega+\nu q^{2}\right)+\beta\left[\mathbf{g}-\mathbf{q}\left(\mathbf{q} \cdot \mathbf{g} / q^{2}\right)\right]}
\end{aligned}
$$

and

$$
\begin{aligned}
&\left\langle\delta c(\mathbf{q}, \omega) \delta \mathbf{u}\left(\mathbf{q}^{\prime}, \omega^{\prime}\right)\right\rangle_{S} \\
&=- \delta\left(\mathbf{q}+\mathbf{q}^{\prime}\right) \delta\left(\omega+\omega^{\prime}\right) \frac{K_{B} T \nu}{8 \pi^{4} \rho} \\
& \times \frac{q^{2}\left(i \omega+D_{0} q^{2}\right)\left[\nabla c-\left(\boldsymbol{\nabla} c \cdot \mathbf{q} / q^{2}\right)\right]}{\left|\left(i \omega+D_{0} q^{2}\right)\left(i \omega+\nu q^{2}\right)+\beta\left[\mathbf{g}-\mathbf{q}\left(\mathbf{q} \cdot \mathbf{g} / q^{2}\right)\right] \cdot \nabla c\right|^{2}} .
\end{aligned}
$$

The denominator of Eqs. (12) and (13) is an even function of $\omega$; therefore, in the evaluation of the integral in Eq. (10) we can neglect odd functions of $\omega$ in the numerator.

By combining Eqs. (10)-(13) and by assuming that $\hat{\mathbf{z}}$ $=\mathbf{g} / g=\boldsymbol{\nabla} c /|\nabla c|$, the current $\mathbf{j}_{f}$ due to nonequilibrium fluctuations is finally determined:

$$
\mathbf{j}_{f}=-\rho D_{f}\left[\boldsymbol{\nabla} c-\beta \mathbf{g}\left(\frac{\partial c}{\partial \mu}\right)_{p, T}\right]
$$

where

$$
\begin{aligned}
D_{f}= & \frac{K_{B} T \nu D_{0}}{8 \pi^{4} \rho} \\
& \times \int \frac{q^{4}\left[\hat{\mathbf{z}}-\mathbf{q}\left(\hat{\mathbf{z}} \cdot \mathbf{q} / q^{2}\right)\right] \cdot \hat{\mathbf{z}}}{\left|\left(i \omega+D_{0} q^{2}\right)\left(i \omega+\nu q^{2}\right)+\beta g \nabla c\left[\hat{\mathbf{z}}-\mathbf{q}\left(\hat{\mathbf{z}} \cdot \mathbf{q} / q^{2}\right)\right] \cdot \hat{\mathbf{z}}\right|^{2}} \\
& \times d \mathbf{q} d \omega .
\end{aligned}
$$

Remarkably, the flow $\mathbf{j}_{f}$ displays the customary dependence of Fick's flow on the driving force $\nabla c$ and on the barodiffusion flow.

Equation (15) can be rewritten by factorizing its denominator as $\left(\omega^{2}+\omega_{+}^{2}\right)\left(\omega^{2}+\omega_{-}^{2}\right)$ and by approximating the two roots as $\omega_{+}^{2}=D_{0}^{2} q^{4}\left\{1+\left[q / q_{R O}(g)\right]^{4}\left[\hat{\mathbf{z}}-\mathbf{q}^{\left(\hat{\mathbf{z}} \cdot \mathbf{q} / q^{2}\right)}\right] \cdot \hat{\mathbf{z}}\right\}^{2}$ and $\omega_{-}^{2}=\nu^{2} q^{4}$. Basically, this approximation holds true for $\nu$ $\gg D$, a condition fulfilled by most binary liquid mixtures; further details are discussed in Ref. [4]:

$$
D_{f}=\frac{K_{B} T}{(2 \pi)^{3} \rho \nu} \int \frac{q^{2}\left[\hat{\mathbf{z}}-\mathbf{q}\left(\hat{\mathbf{z}} \cdot \mathbf{q} / q^{2}\right) \cdot \hat{\mathbf{z}}\right.}{q^{4}+q_{R O}(g)^{4}\left[\hat{\mathbf{z}}-\mathbf{q}\left(\hat{\mathbf{z}} \cdot \mathbf{q} / q^{2}\right)\right] \cdot \hat{\mathbf{z}}} d \mathbf{q},
$$

where

$$
q_{R O}(g)=\sqrt[4]{\frac{g \beta \nabla c}{\nu D_{0}}}
$$

is a roll-off wave vector due to the gravity force [6]. As thoroughly discussed in Ref. [4], $q_{R O}$ is defined as the wave vector marking the transition from the diffusive relaxation of fluctuations at large wave vectors to the gravitational stabilization of fluctuations at smaller ones. At the roll-off wave vector the diffusional and gravitational fluctuation relaxation times coincide.

By introducing polar coordinates Eq. (16) becomes

$$
D_{f}^{Q}=\frac{K_{B} T}{(2 \pi)^{3} \rho \nu} \int_{0}^{Q} d q \int_{0}^{\pi} d \theta \int_{0}^{2 \pi} d \varphi \frac{q^{4} \sin ^{3}(\theta)}{q^{4}+q_{R O}(g)^{4} \sin ^{2}(\theta)} .
$$

The integral on $q$ is divergent. Therefore, we have regularized it by introducing a brute-force cutoff at the arbitrary wave vector $Q$. The evaluation of the integrals in $\varphi$ and $q$ yields

$$
\begin{aligned}
D_{f}^{Q}= & \frac{K_{B} T}{(2 \pi)^{2} \rho \nu} \int_{0}^{\pi} d \theta Q\left[1-\frac{\sqrt{2} \pi}{4} \frac{q_{R O}}{Q} \sqrt{\sin \theta}\right. \\
& \left.+O\left(\frac{q_{R O} \sqrt{\sin \theta}}{Q}\right)^{4}\right] \sin ^{3} \theta .
\end{aligned}
$$

By assuming that $q_{R O}(g) / Q \ll 1$, Eq. (19) becomes

$$
\begin{aligned}
D_{f}^{Q}(g)= & \frac{4}{3} \frac{K_{B} T}{(2 \pi)^{2} \rho \nu} Q-\sqrt{2} I \frac{\pi}{4} \frac{K_{B} T}{(2 \pi)^{2} \rho \nu} q_{R O}(g) \\
& +Q O\left(q_{R O}(g) / Q\right)^{4},
\end{aligned}
$$

where

$$
I=\int_{0}^{\pi} d \theta \sin ^{7 / 2} \theta \approx 1.248599
$$

In the absence of gravity $q_{R O}=0$, and Eq. (20) becomes

$$
D_{f}^{Q}=\frac{2}{3} \frac{K_{B} T}{\pi \rho \nu \Lambda},
$$

where $\Lambda=2 \pi / Q$ can be roughly assumed to be of the order of correlation length of the fluid. It should be noticed that the diffusion coefficient $D_{f}$ of Eq. (22) does not depend on the bare mass diffusion coefficient $D_{0}$ which appeared in the linearized fluctuating hydrodynamic equations (3) and (5).

Quite remarkably, Eq. (22) exhibits the usual StokesEinstein dependence on temperature, viscosity, and correlation length, the proportionality factor being of the right order of magnitude. This result, combined with Eq. (14), strongly suggests that the mass transfer due to fluctuations and Fick's mass flow can be identified. This is a striking result, as it shows for the first time that the diffusive mass transfer is entirely determined by nonequilibrium fluctuations occurring at length scales ranging from the microscopic up to the macroscopic one. 
Although it is physically reasonable to assume that wave vectors larger than a typical molecular one have no meaning, the imposition of a cutoff involves the introduction of an arbitrary length scale, which in turn requires to introduce a microscopic model for the molecular interactions. This necessity can be eliminated by a renormalization of the linearized hydrodynamic equations. The normalization procedure we will discuss in the following closely mirrors the renormalization of mass of quantum field theory [14] and of classical electrodynamics [15]. The overall diffusive mass flow is the superposition of the contribution due to fluctuations $\mathbf{j}_{f}$ and the bare Fickean mass flow:

$$
\begin{aligned}
\mathbf{j}(g) & =\mathbf{j}_{f}^{Q}(g)+\mathbf{j}_{0}^{Q}(g) \\
& =-\rho\left(D_{f}^{Q}(g)+D_{0}^{Q}\right)\left[\boldsymbol{\nabla} c-\beta \mathbf{g}\left(\frac{\partial c}{\partial \mu}\right)_{p, T}\right] .
\end{aligned}
$$

The renormalization condition is

$$
\mathbf{j}(g=0)=-\rho D \nabla c,
$$

where $D$ is the phenomenological diffusion coefficient in the absence of gravity. By combining Eq. (23) and Eq. (24), the renormalization condition can be rewritten as

$$
D_{0}^{Q}=D-D_{f}^{Q}(g=0),
$$

and, by inserting Eqs. (20) and (25) into Eq. (23), the renormalized mass current is

$$
\mathbf{j}_{R}(g)=-\rho D_{R}(g)\left[\boldsymbol{\nabla} c-\beta \mathbf{g}\left(\frac{\partial c}{\partial \mu}\right)_{p, T}\right],
$$

where $D_{R}$ is the renormalized diffusion coefficient

$$
D_{R}(g)=D-\sqrt{2} \frac{\pi}{4} I \frac{K_{B} T}{(2 \pi)^{2} \rho \nu} q_{R O}(g) .
$$

In Eq. (27) the $Q$ dependence has been eliminated by letting $Q \rightarrow \infty$, because $D_{R}$ does not diverge. The microscopic details have been reabsorbed into $D$, the only experimental parameter needed by the theory in order to obtain the value of the diffusion coefficient in the presence of gravity.

Equation (27) shows that the diffusion coefficient is smaller due to the presence of gravity, the additional term having the Stokes-Einstein form, the only length scale involved being $2 \pi / q_{R O}$. This modification of the diffusion coefficient is due to the fact that, in the presence of gravity, fluctuations with wave vector smaller than $q_{R O}$ are stabilized and therefore do not contribute to the mass transfer. Therefore $q_{R O}$ acts as an infrared cutoff for the fluctuations. As the length scales stabilized by gravity are large, the relative correction introduced by gravity is very small, being of the order of $q_{R O} \xi$, where we have assumed a Stokes-Einstein dependence of the phenomenological diffusion coefficient $D$ on the correlation length $\xi$ of the fluid. For an ordinary liquid mixture $q_{R O}$ can be as high as $10^{3} \mathrm{~cm}^{-1}$ and $\xi$ is of the order of $10^{-7} \mathrm{~cm}$. Therefore, the gravitational correction is estimated to be roughly one part in $10^{4}$. In the case of critical fluids and macromolecular solutions, the correlation length can grow very large, thus suggesting that the gravitational correction to the diffusion coefficient can get large. However, in order for our hydrodynamic description to be valid, the correlation length has to be significantly smaller than $1 / q_{R O}$. Therefore, the largest correction to the diffusion coefficient reliably predicted by this description can be roughly estimated to be of the order of $10 \%$. Such a variation in the diffusion coefficient should be observable by performing microgravity experiments on critical fluids.

The authors wish to thank Marzio Giglio for stimulating discussion. This work is partially supported by the Italian Space Agency (ASI).
[1] A. Vailati and M. Giglio, Nature (London) 390, 262 (1997).

[2] D. Brogioli, A. Vailati, and M. Giglio, Phys. Rev. E 61, R1 (2000).

[3] L. D. Landau and E. M. Lifshitz, Fluid Mechanics (Pergamon, New York, 1959).

[4] A. Vailati and M. Giglio, Phys. Rev. E 58, 4361 (1998).

[5] B. M. Law and J. C. Nieuwoudt, Phys. Rev. A 40, 3880 (1989).

[6] P. N. Segré and J. V. Sengers, Physica A 198, 46 (1993).

[7] P. N. Segré, R. W. Gammon, and J. V. Sengers, Phys. Rev. E 47, 1026 (1993).

[8] W. B. Li, P. N. Segré, R. W. Gammon, and J. V. Sengers, Physica A 204, 399 (1994).
[9] A. Vailati and M. Giglio, Phys. Rev. Lett. 77, 1484 (1996).

[10] W. B. Li, K. J. Zhang, J. V. Sengers, R. W. Gammon, and J. M. O. de Zarate, Phys. Rev. Lett. 81, 5580 (1998).

[11] D. A. Weitz, Nature (London) 390, 233 (1997).

[12] C. Cohen, J. W. H. Sutherland, and J. M. Deutch, Phys. Chem. Liq. 2, 213 (1971).

[13] D. Ronis, I. Procaccia, and J. Machta, Phys. Rev. A 22, 714 (1980).

[14] M. Le Bellac, Des Fenomenes Critiques au Champs de Jauge (InterEditions, Paris, 1988).

[15] R. P. Feynman, The Feynman Lectures on Physics (AddisonWesley, Reading, 1964), Vol. 2. 